\title{
A COMPARATIVE STUDY OF THE SHELL OF \\ $\zeta$ TAU AND 48 LIB
}

\author{
A. M. DELPLACE and M. Th. CHAMBON \\ Observatoire de Meudon, France
}

\section{Introduction}

Two unstable shell stars, $\zeta$ Tau and $48 \mathrm{Lib}$, are compared. In the Herman-Rojas (1974) and Rountree-Lesh (1968) classifications, both stars have luminosity class III. These stars show similar features in the variation of the intensities, the profiles, and the radial velocities of the shell lines. An attempt has been made to explain the evolution of these unstable features.

\section{2. $\zeta$ Tauri}

This Be star is a binary system with period 132.91 days. The shell was moving in 1914-17 (Losh, 1932), then quiescent from 1920 to 1952, but since 1955 it has again been in motion. In 1958-59, an expanding shell was found by Hack (1962); in 1958 the radial velocities of the shell lines were $-60 \mathrm{~km} \mathrm{~s}^{-1}$ and in $1959-80 \mathrm{~km} \mathrm{~s}^{-1}$.

This star has been observed at the Haute Provence Observatory with the 193 and $152 \mathrm{~cm}$ telescopes for fifteen years. The dispersions of the spectra are $9.67 \AA \mathrm{mm}^{-1}$ and $12.27 \AA \mathrm{mm}^{-1}$.

\subsection{Outer layers OF THE SHELl}

The radial velocities of the $\mathrm{H} \gamma, \mathrm{H} \delta, \mathrm{H} \varepsilon$ shell lines are shown in Figure 1. The short period is that of the binary system. The long variation is a representation of the radial velocities of the outer layers, but we shall see that the behaviour of the inner layers is more complex. A possible explanation of these variations is the following.

In 1956-59 an expanding shell was driven by rapid internal motions, probably resulting from the instability of the photosphere of the star; when the internal motions stopped, the outer shell fell down on the star until another expanding phase occurred. In our diagram, therefore, the outer shell is oscillating around the $25 \mathrm{~km} \mathrm{~s}^{-1}$ value, which is probably the mean radial velocity value of the star, as it was shown by Losh (1932), Hynek and Struve (1942) and Underhill (1952). We can see that the period of variation of the radial velocities increases with increasing velocity of expansion.

\subsection{INNER LAYERS OF THE SHELL}

The differences between the radial velocities of the inner layers and the outer layers are plotted in Figure 2. For metallic lines, the total excitation energy is defined as the sum of the ionization potential and the excitation potential. 


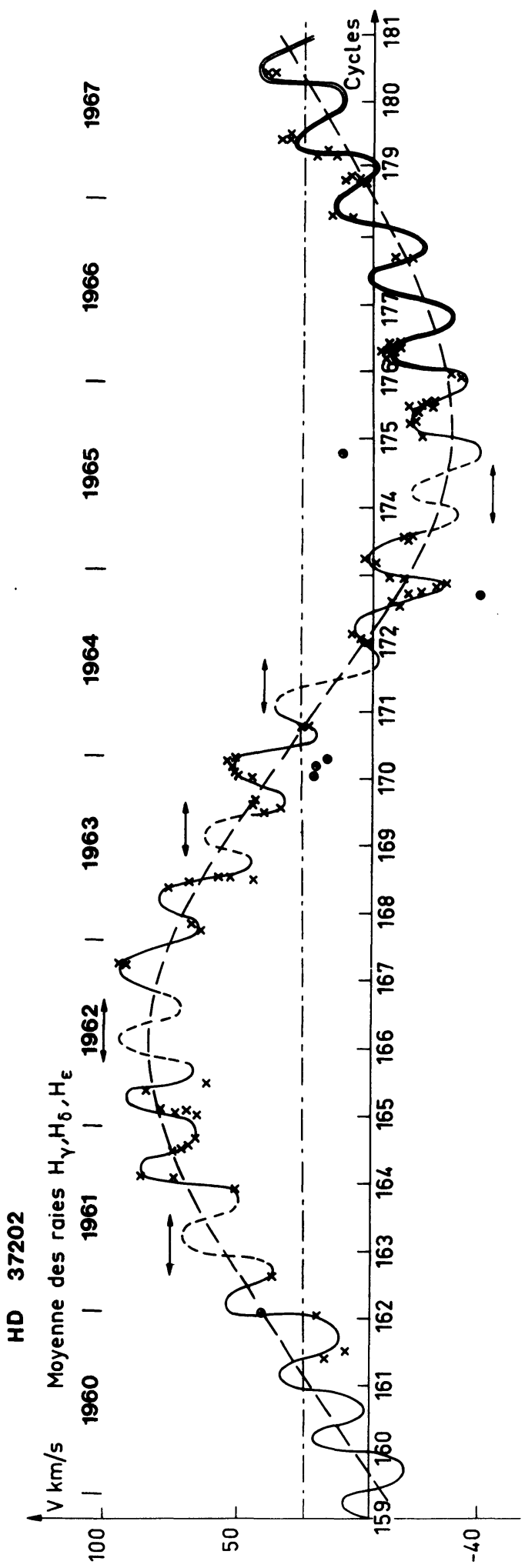




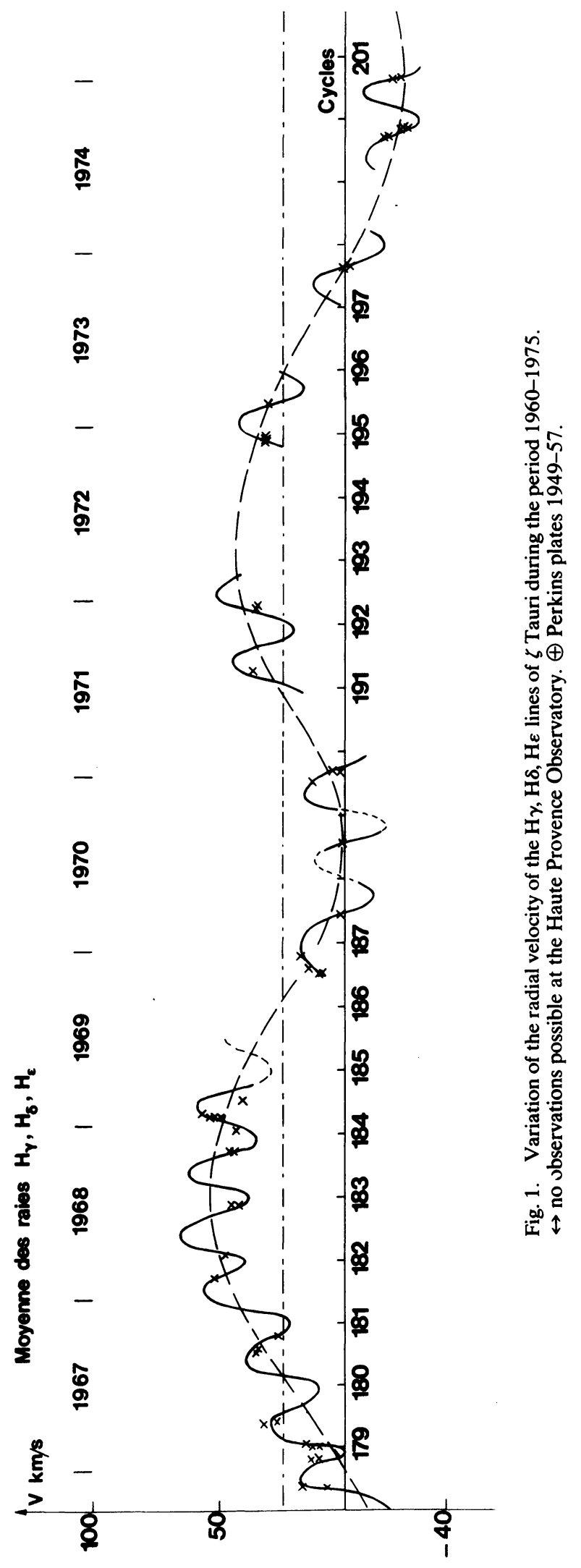



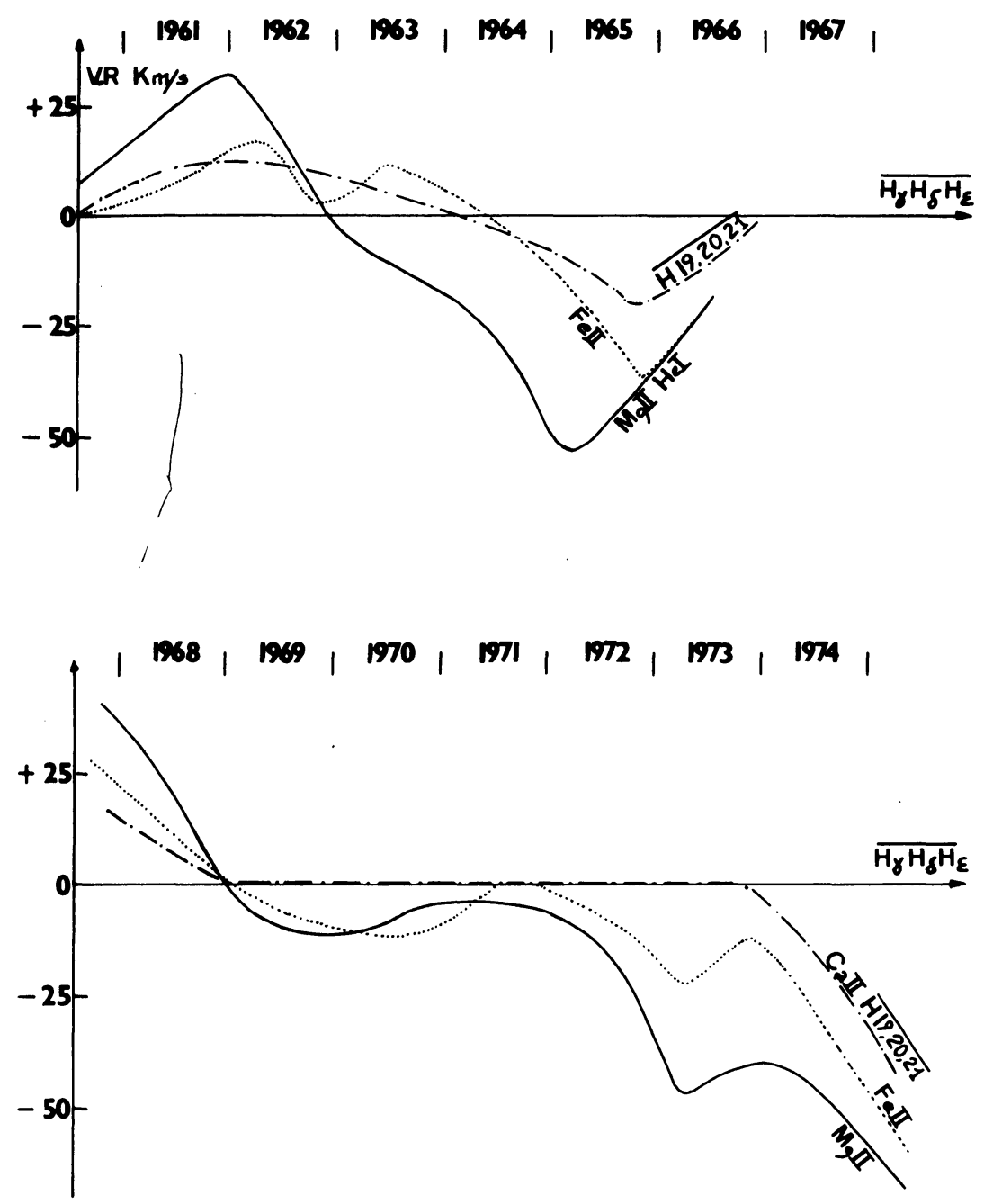

Fig. 2. A schematic representation of the radial velocity curves of the $\mathrm{Mg}$ II and $\mathrm{He}$ I, Fe II, Ca II and $\mathrm{H} 19, \mathrm{H} 20$, and $\mathrm{H} 21$ lines in comparison with the radial velocity of the $\mathrm{H} \gamma, \mathrm{H} \delta$, and $\mathrm{H} \varepsilon$ lines.

We assume that with increasing total excitation energy, the depth in the shell at which the line is formed increases.

(a) The radial velocities of the line increase for higher total excitation energy. The radial velocity of the $\mathrm{Mg}$ II line at $\lambda 4481 \AA$ is the most important. The radial velocities of the metallic lines (Fe II, Cr II, Ti II, Ni II) are more important than the radial velocities of the Ca II lines at $\lambda \lambda 3933 \AA$ and $3968 \AA$. These Ca II lines have about the same radial velocities as the $\mathrm{H} 19, \mathrm{H} 20$, and $\mathrm{H} 21$ lines.

(b) The expanding motions are much bigger and longer in the inner layers. They are transmitted to the outer layers but with a damped and lagging manner (the radial velocity curve of the hydrogen lines is shifted back, about several months, in 
comparison to the radial velocity curve of the $\mathrm{Mg}$ II line). Thus at the end of 1962 , the outer layers were falling back down on the star but some expanding motions occurred in the inner layers because a satellite component, shifted to shorter wavelengths, appears in the $\mathrm{He}$ I and $\mathrm{Mg}$ II lines at about $-60 \mathrm{~km} \mathrm{~s}^{-1}$. In 1964, the expanding motions of the inner layers were increasing and at the end of 1964 the whole shell was moving out (Delplace, 1970). At the end of 1972, the outer shell was stationary but the radial velocities of $\mathrm{He} \mathrm{I}$ and $\mathrm{Mg}$ II became negative. In 1974, the expanding motions in the inner layers were more important and the whole shell moved out again.

\section{48 Librae}

This shell star has been studied particularly by Underhill (1966), Faraggiana (1969, 1971), and Geuverink (1970). From 1904-31 it was considered as a fast rotator surrounded by a faint hydrogen shell. Since 1932-35, the shell has become variable. The radial velocities of the shell lines given by Underhill, Faraggiana, and our own measurements on the spectra taken at the Haute Provence Observatory since 1968 are considered and the schematic curve of these variations shown in Figure 3. The

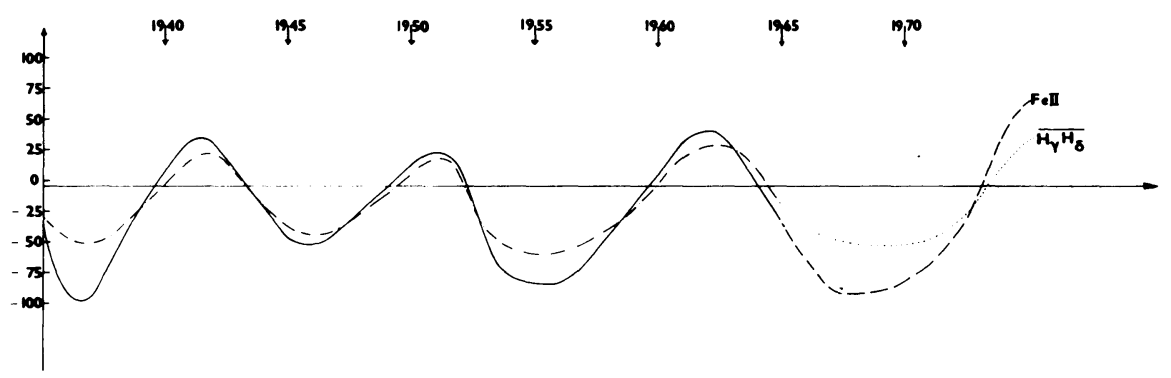

Fig. 3. A schematic representation of the radial velocity curve of the $\mathrm{H} \gamma, \mathrm{H} \delta$ and $\mathrm{Fe}$ Il lines of $48 \mathrm{Librae}$ during the period 1935-75.

radial velocity of the star is about $-5 \mathrm{~km} \mathrm{~s}^{-1}$. As for $\zeta$ Tauri, the radial velocity variations can be interpreted as a sequence of the expanding phases followed by the infalling of material. The time of the oscillation increases with increasing amplitude of the variation. For 48 Librae the infalling material phases are short (about 3 or 4 years).

In the inner layers the motions are much more important than in the outer layers. Faraggiana (1969) showed that the radial velocity of the $\mathrm{H} \alpha$ shell line was $-30 \mathrm{~km} \mathrm{~s}^{-1}$ in $1957-58$ when the radial velocity of the $\mathrm{He} \mathrm{I}$ and $\mathrm{Mg}$ II lines was $-100 \mathrm{~km} \mathrm{~s}^{-1}$.

The difference between the radial velocities of the inner and outer layers is shown in Figure 4. The amplitude of the motions increases for higher total excitation potential. The radial velocity curve of the Ca II lines is shifted several months back in comparison to the Mg II radial velocity curve. In 1974, when the outer shell was 


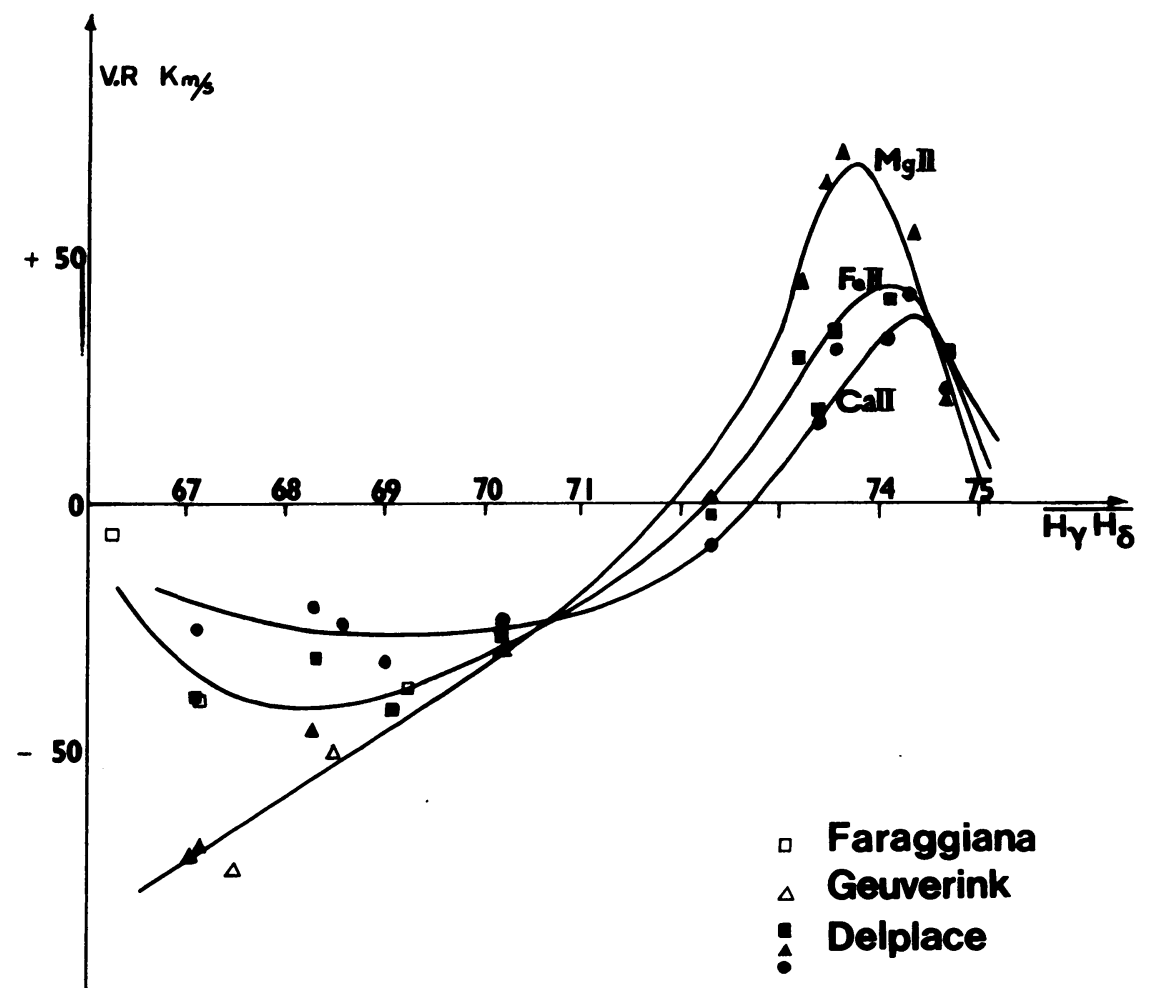

Fig. 4. A schematic representation of the radial velocity curve of the $\mathrm{Mg} \mathrm{II}, \mathrm{Fe}$ II, and $\mathrm{Ca}$ II lines in comparison with the radial velocity of the $\mathrm{H} \gamma$ and $\mathrm{H} \delta$ lines.

falling back on the star, a new expanding phase began because the lines which are formed in the inner layers are double, one component being shifted to shorter wavelengths.

\section{Conclusion}

The behaviour of the shells of $\zeta$ Tauri and 48 Librae is similar. The amplitude of the motion increases for higher total excitation potential. The period of the oscillation of the outer layers increases with increasing velocity of expansion. The internal motions in the shell give rise to a damped oscillation of the outer layers.

\section{References}

Delplace, A. M.: 1970, Astron. Astrophys. 7, 68.

Faraggiana, R.: 1969, Astron. Astrophys. 2, 162. 
Faraggiana, R.: 1971, Astrophys. Letters 8, 45.

Geuverink, H. G.: 1970, Astron. Astrophys. 5, 341.

Hack, M.: 1962, Publ. Astron. Soc. Pacific 74, 78.

Herman, R.: 1974, private communication.

Hynek, J. A. and Struve, O.: 1942, Astrophys. J. 96, 425.

Losh, H. M.: 1932, Publ. Obs. Univ. Michigan 4, 1.

Rountree-Lesh, J.: 1968, Astrophys. J. Suppl. 16, 371.

Underhill, A. B.: 1952, Publ. Dominion Astrophys. Obs. 9, 138.

Underhill, A. B.: 1966, The Early Type Stars, D. Reidel Publ. Co., Dordrecht, p. 238. 